\title{
Perfect Chemotherapy of Uterine Malignancy Followed by Normal Pregnancy
}

\section{Kazuo Maeda*}

Department of Obstetrics and Gynecology (Emeritus), Tottori University Medical School, Yonago, Japan

The choriocarcinoma is a uterine malignant neolasia characterized by postmolar development accompanied with general metastases, and the patient died by brain hemorrhage of brain metastasis within 2 years after the total hysterectomy in old times. The author was enthusiastic to treat the malignancy as a systemic disease instead of a local malignancy within the uterus with MTX chemotherapy in 1960s, to which vhoriocarcinoma was highly sensitive and even brain metastasis disappeared by the complete remission after MTX chemotherapy. Invasive mole was another trophoblastic tumor localized in the myometrium but its sensitivity to MTX was lower than choriocarcinoma, because the molar tissue was more highly differentiated than choriocarcinoma. A surgery was the most effective in the invasive mole, where it was ultrasonically diagnosed by its villous structure in the myometrium. Since the primary choriocarcinoma focus was usually located in the uterus, it was treated by MTX chemotherapy prior to hysterectomy at the same time with the distant metastasis. That was the primary chemotherapy, which preserved uterus, promising future pregnancy and child birth, if the primary focuses disappeared by the MTX chemotherapy. Some choriocarcinoma patients received the primary chemotherapy followed by the recovery of fertility and normal pregnancy. The MTX chemotherapy of choriocarcinoma was provided usually by intensive regimen, where $0.4-0.5 \mathrm{mg} / \mathrm{kg}$ MTX was injected intramuscularly or by drip infusion for 3-4 days in a week, followed by 3-6 mg Leucovorin after MTX administration. Actinomycin-D was usually combined with MTX. The chemotherapy was monitored by the HCG in the blood or urine, where the chemotherapy course was repeated every week until the disappearance of HCG [1]. Another monitor to detect choriocarcinoma sensitivity was the ultrasonic pulsed Doppler peripheral blood floe resistance of the tumor mass, i.e. resistance index and pulsatility index of tumor vascular pulsed Doppler showed clear elevation after starting the MTX chemotherapy. That may show the shrinkage of tumor mass caused by the chemotherapy. A non-sensitive trophoblastic tumor.e.g. invasive mole, did not show the elevation of RI and PI by the chemotherapy.

Prophylactic chemotherapy to prevent postmolar choriocarcinoma was tried in a controlled study in Kyushu University by us, looking the therapeutic effect of MTX on choriocarcinoma in 1968 [2]. Postmolar women were divided in two groups, who received orally $10 \mathrm{mg}$ MTX for a week in the chemotherapy group, and the other received no chemotherapy. Since the chemotherapy was prolonged until the disappearance of urinary HCG, two persistent trophoblastic disease patients after complete mole received MTX for more than $200 \mathrm{mg}$ in total. In the results, no choriocarcinoma developed in the chemotherapy group, while some choriocarcinoma developed in non-chemotherapy group with significant difference. Afterwards, choriocarcinoma was very rare in Japan.

We, medical staffs, will be very happy, if the primary chemotherapy and prophylactic chemotherapy are successful in the other malignancy as has done in choriocarcinoma.

\section{References}

1. Tow WS, Cheng WC (1970) Treatment of choriocarcinoma. Br Med J 2: 764.

2. Koga K, Maeda K (1968) Prophyractic chemotherapy with amethopterin for prevention of choriocarcinoma following removal of hydatidiform mole. Amer $\mathrm{J}$ Obstet.Gynecol 100: 270-275.
*Corresponding author: Kazuo Maeda, Department of Obstetrics and Gynecology (Emeritus),Tottori University Medical School, Yonago, Japan, 3-125, Nadamachi, Yonago, Tottoriken, 6830835, Japan, Tel: 81-859-22-6856; Fax: 81-859-22-6856; E-mail: maeda@mocha.ocn.ne.jp

Received November 22, 2013; Accepted November 23, 2013; Published November 28, 2013

Citation: Maeda K (2013) Perfect Chemotherapy of Uterine Malignancy Followed by Normal Pregnancy. J Health Med Informat. 5: e118. doi:10.4172/2157$7420.1000 \mathrm{e} 118$

Copyright: ( 2013 Maeda K. This is an open-access article distributed under the terms of the Creative Commons Attribution License, which permits unrestricted use, distribution, and reproduction in any medium, provided the original author and source are credited. 